\title{
Phenotypic expansion illuminates multilocus pathogenic variation
}

\author{
Ender Karaca, MD 1,11,13, Jennifer E. Posey, MD, PhD ${ }^{1,13}$, Zeynep Coban Akdemir, PhD ${ }^{1}$, \\ Davut Pehlivan, MD1, Tamar Harel, MD, PhD², Shalini N. Jhangiani, MS³, Yavuz Bayram, MD ${ }^{1,12}$, \\ Xiaofei Song, $\mathrm{BS}^{1}$, Vahid Bahrambeigi, $\mathrm{MS}^{1,4}$, Ozge Ozalp Yuregir, MD, PhD ${ }^{5}$, Sevcan Bozdogan, $\mathrm{MD}^{6}$, \\ Gozde Yesil, MD, Sedat Isikay, MD ${ }^{8}$, Donna Muzny, MS ${ }^{3}$, Richard A. Gibbs, $\mathrm{PhD}^{1,3}$ and \\ James R. Lupski, MD, PhD, DSc (hon) $)^{1,3,9,10}$
}

\begin{abstract}
Purpose: Multilocus variation-pathogenic variants in two or more disease genes-can potentially explain the underlying genetic basis for apparent phenotypic expansion in cases for which the observed clinical features extend beyond those reported in association with a "known" disease gene.

Methods: Analyses focused on 106 patients, 19 for whom apparent phenotypic expansion was previously attributed to variation at known disease genes. We performed a retrospective computational reanalysis of whole-exome sequencing data using stringent Variant Call File filtering criteria to determine whether molecular diagnoses involving additional disease loci might explain the observed expanded phenotypes.
\end{abstract}

Results: Multilocus variation was identified in 31.6\% (6/19) of families with phenotypic expansion and $2.3 \%$ (2/87) without phenotypic expansion. Intrafamilial clinical variability within two families was explained by multilocus variation identified in the more severely affected sibling.

Conclusion: Our findings underscore the role of multiple rare variants at different loci in the etiology of genetically and clinically heterogeneous cohorts. Intrafamilial phenotypic and genotypic variability allowed a dissection of genotype-phenotype relationships in two families. Our data emphasize the critical role of the clinician in diagnostic genomic analyses and demonstrate that apparent phenotypic expansion may represent blended phenotypes resulting from pathogenic variation at more than one locus.

Genet Med advance online publication 26 April 2018

Key Words: distinct/overlapping blended phenotypes; multilocus variation; neurodevelopmental disorder; personal genomes; phenotypic expansion of Mendelizing disease traits

\section{INTRODUCTION}

The diagnostic path leading from clinical evaluation to genetic diagnosis is not always direct, and physicians have historically relied on hallmark patterns of history and physical examination findings, or phenotypic features, to formulate a differential diagnosis and guide clinical investigation. However, even "typical" clinical presentations can be misleading, as illustrated by early reports of Fitzsimmons syndrome describing six individuals with a seemingly unique combination of spastic paraplegia, skeletal anomalies, dysarthria, and mild developmental delay. ${ }^{1-3}$ Whole-exome sequencing (WES) ultimately demonstrated that despite the consistent set of shared phenotypic features, a single genetic locus did not explain the full phenotypic spectrum of the condition. ${ }^{4}$ Multiple segregating pathogenic alleles at single $e^{5,6}$ or multiple ${ }^{7,8}$ loci in a family or clan can challenge both clinical and molecular diagnosis.
The occurrence of dual, or multiple, molecular diagnoses in an individual is not new, and reports dating back to the 1960s describe individuals with both hemolytic anemia due to glucose-6-phosphate dehydrogenase deficiency (OMIM 300908) and thalassemia minor, $\beta$-thalassemia, or sickle cell trait. ${ }^{9,10}$ The true frequency of multiple molecular diagnoses has only begun to emerge with the availability of genome-wide investigations such as chromosome microarray analysis ${ }^{11}$ and WES; larger-scale analyses of diagnostic WES in a clinical setting have revealed that 3.2 to $7.2 \%$ of unselected diagnostic WES cases have two or more molecular diagnoses contributing to their observed clinical phenotype ${ }^{8,12-17}$ This rate can be even higher, $12 \%(5 / 41)$, in selected cohorts. ${ }^{18}$ From a clinical standpoint, recognition of multiple molecular diagnoses can have important implications for both treatment and estimates of familial recurrence risk. ${ }^{8}$

\footnotetext{
${ }^{1}$ Department of Molecular and Human Genetics, Baylor College of Medicine, Houston, Texas, USA; ${ }^{2}$ Department of Genetic and Metabolic Diseases, Hadassah-Hebrew University Medical Center, Jerusalem, Israel; ${ }^{3}$ Human Genome Sequencing Center, Baylor College of Medicine, Houston, Texas, USA; ${ }^{4}$ Graduate Program in Diagnostic Genetics, School of Health Professions, University of Texas MD Anderson Cancer Center, Houston, Texas, USA; ${ }^{5}$ Genetic Diagnosis Center, University of Health Sciences, City Hospital, Adana, Turkey; ${ }^{6}$ Department of Medical Genetics, Cukurova University Faculty of Medicine, Adana, Turkey; ${ }^{7}$ Department of Medical Genetics, Bezmialem University, Istanbul, Turkey; ${ }^{8}$ Department of Physiotherapy and Rehabilitation, Hasan Kalyoncu University, School of Health Sciences, Gaziantep, Turkey; ${ }^{9}$ Department of Pediatrics, Baylor College of Medicine, Houston, Texas, USA; ${ }^{10}$ Texas Children's Hospital, Houston, Texas, USA. Correspondence: Jennifer E. Posey or James R. Lupski (Jennifer.Posey@bcm.edu or jlupski@bcm.edu)

${ }^{11}$ Current address: Department of Genetics, University of Alabama at Birmingham, Birmingham, Alabama, USA.

${ }^{12}$ Current address: Department of Genetics and Genomic Sciences, Icahn School of Medicine at Mount Sinai, New York, New York, USA.

${ }^{13}$ The first two authors are co-first authors.

Submitted 20 August 2017; accepted 24 January 2018; advance online publication 26 April 2018. doi:10.1038/gim.2018.33
} 
Genome-wide analyses have been instrumental for identifying molecular diagnoses in the setting of both atypical presentations of well-characterized syndromes, ${ }^{7}$ as well as cases for which the set of observed phenotypic features extended beyond those previously reported in association with a particular locus, termed phenotypic expansion. ${ }^{19}$ Examples of phenotypic expansion attributed to previously known disease loci abound, with a striking 18.2\% (174/956) of discoveries reported by the National Institutes of Healthfunded Centers for Mendelian Genomics (CMG) representing apparent phenotypic expansion. ${ }^{19}$ Given the relative frequency of both phenotypic expansion and multiple molecular diagnoses, we explored the hypothesis that cases of apparent phenotypic expansion may sometimes represent a blended phenotype resulting from variation at more than one locus. We tested this hypothesis using extensive phenotyping and personal genome data (WES) from 106 unrelated subjects from a well-characterized neurodevelopmental cohort, including 19 families with apparent phenotypic expansion that had previously been attributed to variation at a known disease gene. $^{20} \mathrm{~A}$ high prevalence of identity-by-descent enabled a focus on phenotypic and genotypic variability among siblings with shared genetic backgrounds. ${ }^{20}$

\section{MATERIALS AND METHODS}

\section{Patients}

Patients provided informed consent, including consent to publish photographs, under the Baylor Hopkins Center for Mendelian Genomics protocol H-29697 approved by the Baylor College of Medicine Institutional Review Board.

Please see Supplementary Materials and Methods online for details of clinical cohort, analysis approach, and exome coverage metrics.

\section{RESULTS}

We investigated the hypothesis that an observed clinical phenotype, initially interpreted as phenotypic expansion associated with pathogenic variation at one known disease gene locus, may actually represent a blended phenotype due to multilocus variation. We compared the frequency of multilocus variation between cases with and without apparent phenotypic expansion, using a preexisting, well-phenotyped cohort for which WES had previously demonstrated a high rate of molecular diagnoses (84.4\%, 108/128 families). ${ }^{20}$ Of 108 families for whom a molecular diagnosis was identified, 19 families were classified as having phenotypic expansion due to the presence of observed clinical features that extended beyond those previously reported in association with variation at the identified locus (Table 1). ${ }^{20}$ In the present pilot study, we performed a reanalysis of exome variant data from 106 of these 108 families; 2 families were removed from analysis as the exome variant data were previously generated at another institution using a different platform and capture.

Using an objective, very stringent set of Variant Call File filtering criteria to parse the personal genome sequencing data, followed by manual review, we identified a set of rare variants predicted to be damaging to protein function in 51 loci in 37 families for which previously reported disease-gene associations indicated a phenotypic overlap with the proband's observed clinical features. From this set of variants, candidate etiologic variants were selected if they followed expected segregation patterns in affected and unaffected available relatives and were confirmed through independent orthogonal dideoxy Sanger sequencing. This final analysis step identified variants in 10 loci in 8 of 106 (7.5\%) families (Table 1, Supplementary Table S1 online). Notably, the originally reported variants/loci were parsed and not identified in $60.2 \%(65 / 108)$ of families using these strict filtering criteria, which increase the specificity of personal genome analysis but decrease the sensitivity, highlighting the limitations of utilizing a purely computational approach to vcf data filtration prior to manual variant review of individual genomes within a large data set. Variants at eight loci were identified in six families $(6 / 19,31.6 \%)$ classified as having phenotypic expansion, and variants at two loci were identified in two families $(2 / 87,2.3 \%)$ without phenotypic expansion, demonstrating that apparent phenotypic expansion is more frequently associated with multilocus variation (two-tailed Fisher's exact $p=0.0004)$. Notably, this analysis demonstrated a second variant in HOU1960 (BAB4892, C2CD3) that was interpreted to explain the full phenotype of the proband; because the phenotypic contribution of the originally identified IQSEC2 variant was less clear, this family was not considered to have multilocus variation. Candidate novel disease genes were identified in two additional families previously classified as having phenotypic expansion, raising the potential rate of multilocus variation in cases of apparent phenotypic expansion to $42.1 \%(8 / 19)$; these candidate genes are discussed in further detail below. Taken together with the three cases of multilocus variation described in the original study (listed in Table 1$)^{20}$, the overall rate of multilocus variation for this neurodevelopmental cohort is $12.0 \%(13 / 108)$.

In the phenotypic expansion cohort, variants at a second or third locus involving known disease genes were observed in 6 families $(31.6 \%, 6 / 19)$, and included COL3A1 (HOU1341), AMPD2 (HOU1838), GPR126 (HOU1857), TUBA1A (HOU1965), ALG8 and CLN5 (HOU2293), and MED17 and SPG7 (HOU2337, Table 1). In each case, variation at the second and sometimes third locus provided a molecular etiology for the expanded objectively determined phenotype (Table 1). In the nonphenotypic expansion cohort, variants at a second locus related to the observed clinical phenotype were identified in two families: GNAO1 (HOU2092) and ATRX (HOU2442). Unlike the phenotypic expansion cohort, the initially reported (Table 1) variants identified in the nonphenotypic expansion group were interpreted as causative of all phenotypic features in each case. Objective reanalysis of variant data demonstrated that each of these cases had an additional rare variant in a second gene associated with a subset of the observed clinical features. Notably, one case (HOU2268, BAB5990) with macrocephaly 


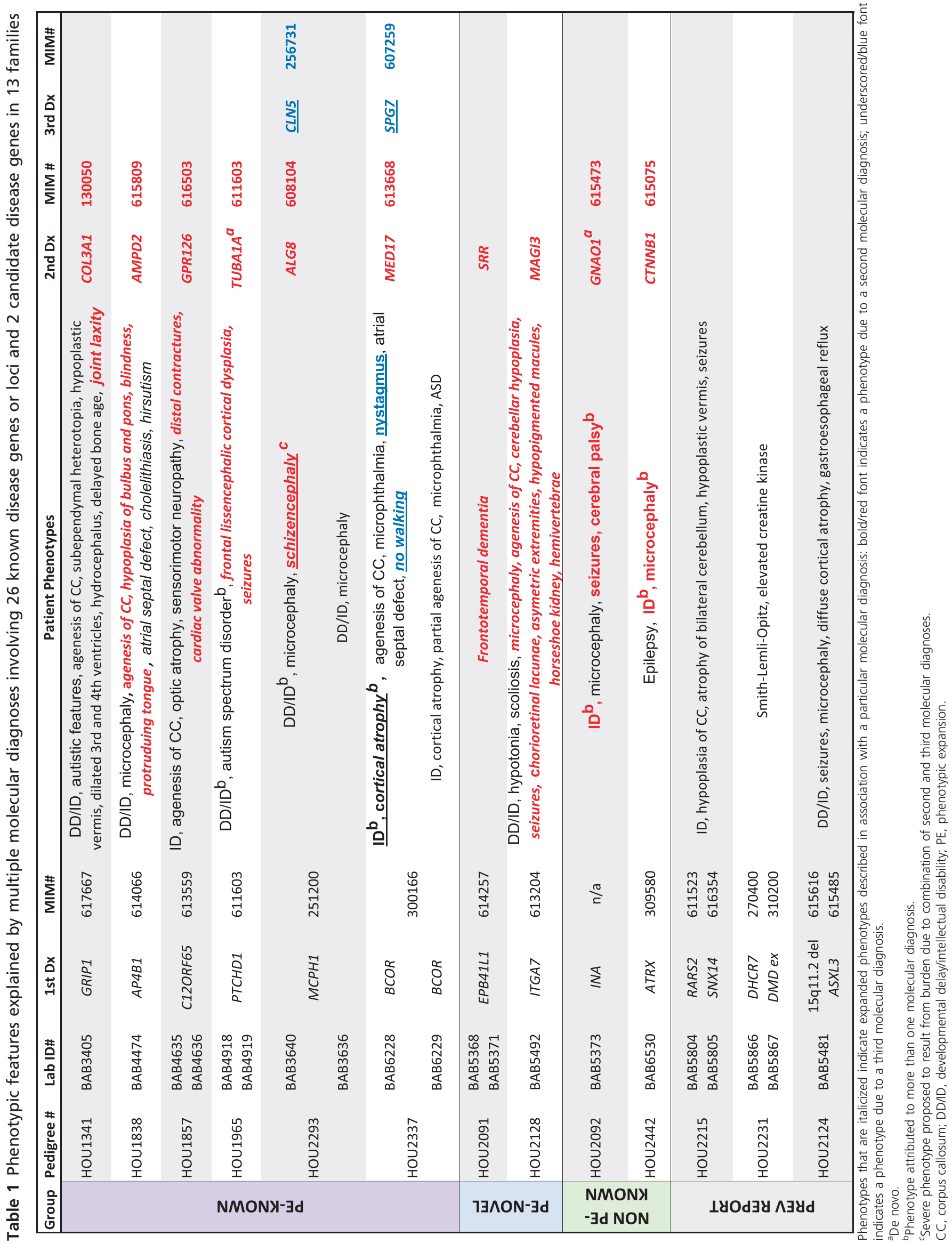


with extended subarachnoid spaces, a thin corpus callosum, and hypotonia was previously reported as having biallelic variation in ZNF423. ${ }^{20}$ In this reanalysis, a de novo $A H D C 1$ frameshifting variant was identified and because of the comprehensive phenotypic match with $A H D C 1$ pathogenic variation, ${ }^{21,22}$ the proband's reported clinical diagnosis of Joubert syndrome (OMIM 614844) was considered less likely. This case was not classified as having multilocus variation in the present study.

\section{Intrafamilial variability allows a dissection of genotype- phenotype correlation}

Two families initially classified as having phenotypic expansion in particular demonstrated phenotypic variability between affected siblings. In pedigree HOU2293, the proband (BAB3640) was reported to have intellectual disability, neurodevelopmental delay, microcephaly, and schizencephaly; this individual's sister (BAB3636), however, had intellectual disability, neurodevelopmental delay, and microcephaly without schizencephaly (Figure 1). Initial WES analysis demonstrated a molecular diagnosis of primary microcephaly (OMIM 251200) due to biallelic pathogenic, frameshifting variants in $\mathrm{MCPH1}$ in both siblings. ${ }^{20}$ Reanalysis of WES data in the proband and sibling demonstrated additional molecular diagnoses of congenital disorder of glycosylation type Ih (OMIM 608104) and neuronal ceroid lipofuscinosis 5 (OMIM 256731) due to rare biallelic

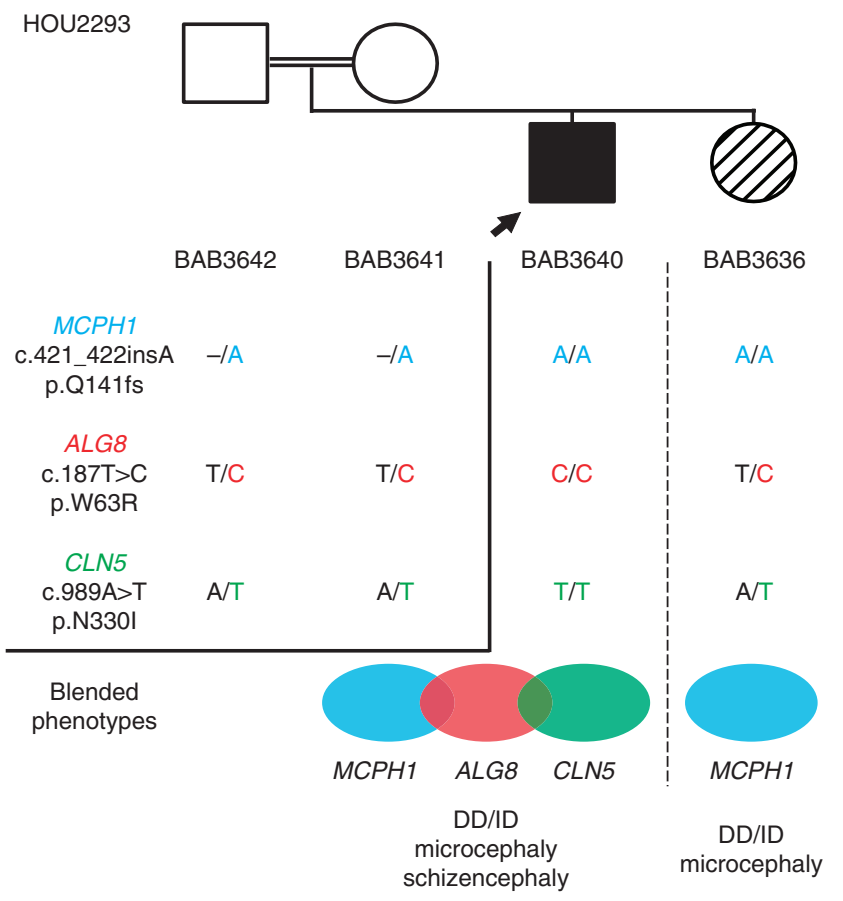

Figure 1 Intrafamilial genotypic and phenotypic variability in HOU2293. Pedigree structure of family HOU2293. Both affected siblings share a homozygous variant in MCPH1; however, the proband (BAB3640) has additional homozygous variants in ALG8 and CLN5, resulting in a more severe blended phenotype. DD/ID, developmental delay/intellectual disability. variants in ALG8 and CLN5 (Figure 1) present only in the more affected proband; notably, none of the three unaffected siblings were homozygous for these variants. Pathogenic variation at both of these loci has been associated with structural brain anomalies including brain atrophy,,$^{23,24}$ and we propose that the additional mutational burden conferred by rare variation at ALG8 and CLN5 led to the schizencephaly phenotype identified in the more severely affected proband.

In pedigree HOU2337, the proband (BAB6228) was reported to have cortical atrophy, agenesis of the corpus callosum, microphthalmia, and severe neurodevelopmental delay characterized by the inability to walk, even with support. This individual's brother (BAB6229) had a milder phenotype with cortical atrophy, partial agenesis of the corpus callosum, and microphthalmia; despite neurodevelopmental delay, he was eventually able to walk independently. A maternally inherited hemizygous variant in $B C O R$ consistent with a molecular diagnosis of syndromic microphthalmia 2 (OMIM 300166) was identified in both siblings (Figure 2). ${ }^{20}$ Reanalysis of WES data further demonstrated biallelic variants in MED17 and SPG7, consistent with molecular diagnoses of postnatal progressive microcephaly with seizures and brain atrophy (OMIM 613668) and autosomal recessive spastic paraplegia (OMIM 607259), in the more severely affected proband only (Figure 2). Although the proband does not demonstrate the full set of features for each of these

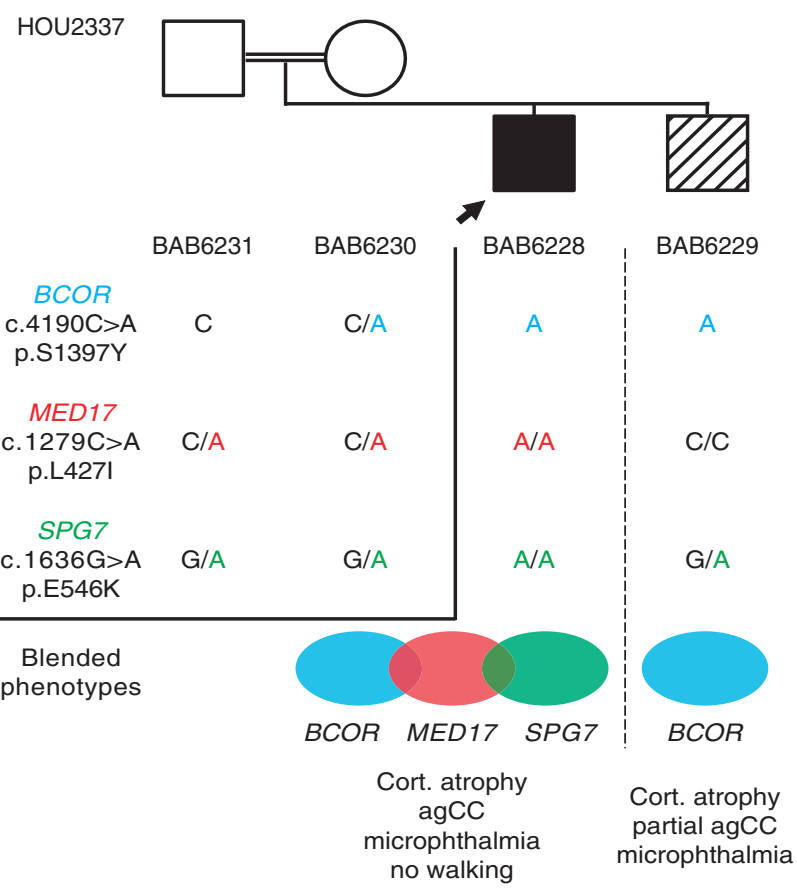

Figure 2 Intrafamilial genotypic and phenotypic variability in HOU2337. Pedigree structure of family HOU2337. Both affected siblings share a hemizygous variant in BCOR; the proband (BAB6228) has additional homozygous variants in MED17 and SPG7, resulting in a more severe phenotype due to the additional mutational burden. agCC, agenesis of the corpus callosum; Cort, cortical. 
diagnoses, both loci were hypothesized to provide genotypic correlation in the form of additional mutational burden to the more severe neurodevelopmental phenotype observed in the proband. Sibling pairs demonstrating phenotypic variability allow a true functional analysis of shared and private variation at genomic loci associated with Mendelian disease and support the contention that all affected individuals in a family or clan should be thoroughly investigated from a clinical phenotyping perspective.

\section{Multilocus variation involving candidate novel disease genes}

Variants at a second locus suggesting a potentially novel disease gene were observed in two families $(10.5 \%, 2 / 19$, Table 1$)$. The proband in HOU2091 has frontotemporal dementia. Initial WES analysis revealed a heterozygous variant in EPB41L1 associated with mental retardation, autosomal dominant 11 (OMIM 614257). On reanalysis, a rare heterozygous variant at a second locus, $S R R$ encoding serine racemase, was identified in the affected proband (BAB5368), as well as the affected mother (BAB5369) and affected maternal uncle (BAB5371). SRR is expressed in mammalian brain, where it converts L-serine to Dserine, a relevant compound for glutamatergic neurotransmission. Studies of Srr knockout mice have demonstrated both a reduction of D-serine in the brain and a reduction of $\mathrm{N}$-methylD-aspartate-induced neurotoxicity, the latter of which has been proposed to be a potential mechanism for the development of the dementia observed in Alzheimer disease. ${ }^{25}$ Based on the known function of serine racemase and its expression pattern, we propose that the identified $S R R$ variant may function in a gain-of-function manner, resulting in a predisposition to frontotemporal dementia.

The proband of family HOU2128 has microcephaly, agenesis of the corpus callosum, cerebellar hypoplasia, seizures, horseshoe kidney, scoliosis, hemivertebrae, asymmetric extremities, and hypopigmented skin macules. This individual was initially found to have a rare and potentially deleterious variant in ITGA7, conferring a molecular diagnosis of congenital muscular dystrophy (OMIM 613204, Table 1). Reanalysis of the WES data demonstrated variation in MAGI3, encoding membrane-associated guanylate kinase with inverted structure 3, and not previously associated with
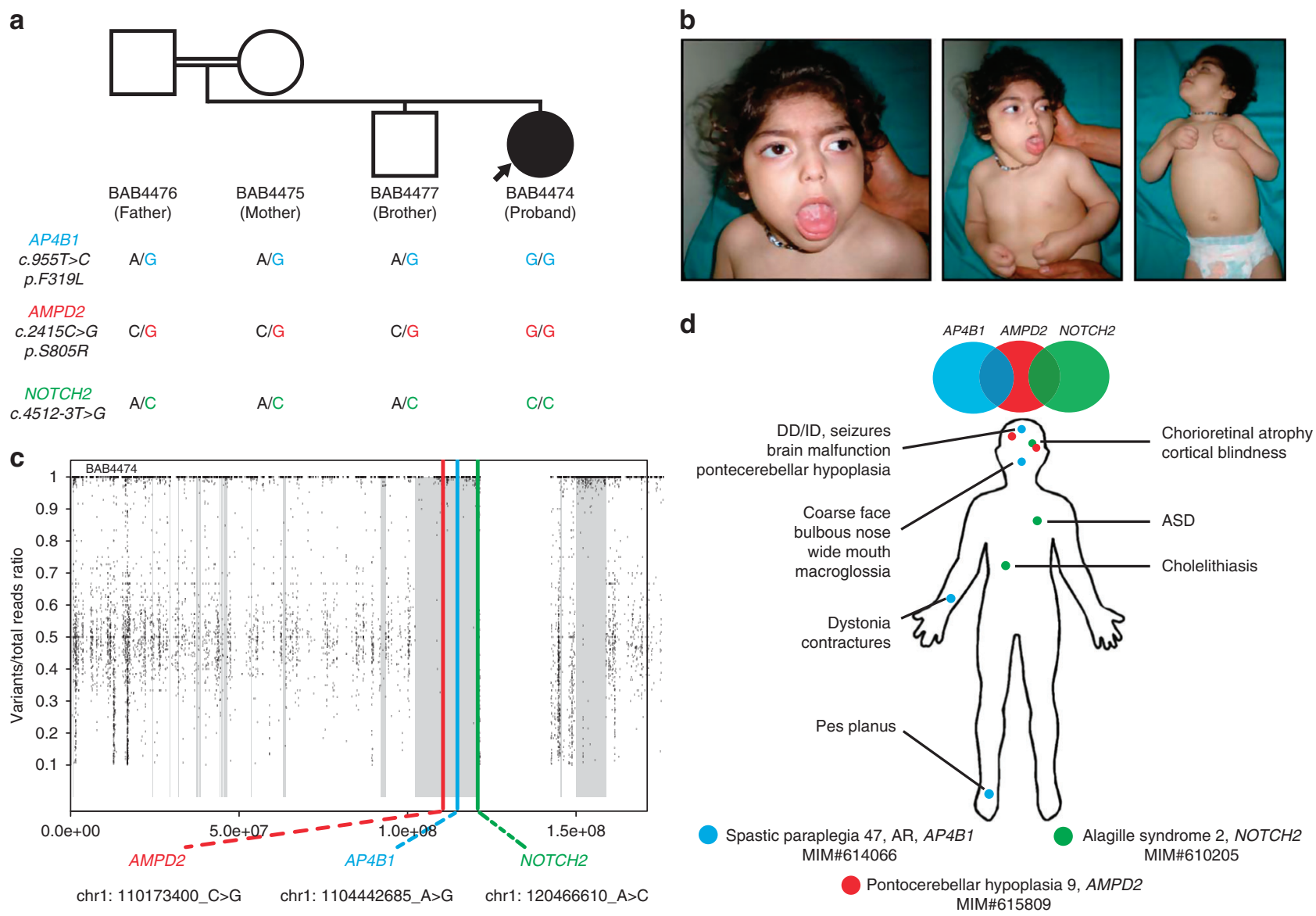

Figure 3 Absence of heterozygosity mediates mutational burden at a single locus. (a) Pedigree structure of family HOU1838 and segregation of the variants identified in AMPD2, AP4B1 and NOTCH2. (b) Images of proband (BAB4474) demonstrate narrow forehead, esotropia of the left eye, short nose and protruding tongue. (c) B-allele frequency calculated from exome variant data demonstrates several regions of absence of heterozygosity $(\mathrm{AOH})$, marked by gray zones. Variants in AMPD2, AP4B1, and NOTCH2 are located within the same region of AOH. (d) Blended phenotypic features and associated syndromes are presented. ASD, atrial septal defect; DD/ID, developmental delay/intellectual disability. 
disease. The membrane associated guanylate kinase proteins, MAGI1, MAGI2, and MAGI3, are expressed in the mammalian brain. ${ }^{26}$ MAGI2 in particular has been described as causative of mental retardation, autosomal dominant 10 (OMIM 614256) in humans. ${ }^{27}$ Disruption of MAGI2 in humans can lead to a seizure phenotype, as atypical 7q11.23 deletions of the Williams-Beuren syndrome (OMIM 194050) region that extend more distally to include MAGI2 are associated with seizures and infantile spasms. ${ }^{28}$ MAGI3 enhances the ability of PTEN to regulate AKT kinase activity, particularly under conditions of low PTEN expression, ${ }^{29}$ and also plays a role in efficient trafficking of TGF- $\alpha$ to the cell surface in polarized epithelial cells. ${ }^{26}$ Based on the expression pattern and function of MAGI3, and the known disease association of MAGI2, we propose that MAGI3 may be a novel candidate gene associated with structural and functional neurologic abnormalities and also potentially with asymmetric extremities.

\section{Absence of heterozygosity-mediated mutation burden}

One of the advantages of the cohort chosen for this analysis is the high rate of reported consanguinity, enabling pathogenic variants to be reduced to homozygosity within a single generation, $^{30}$ and facilitating study of recessive disease, particularly in sibling pairs sharing a similar genetic background. Considering the 8 probands with a second and a
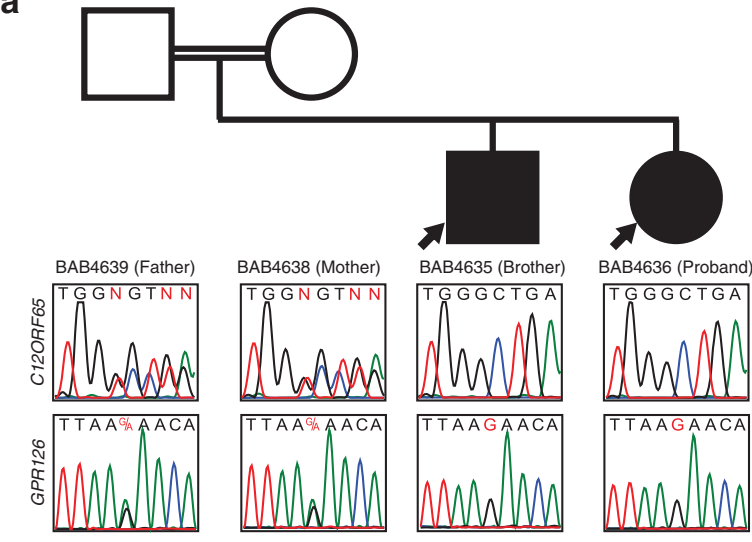

C
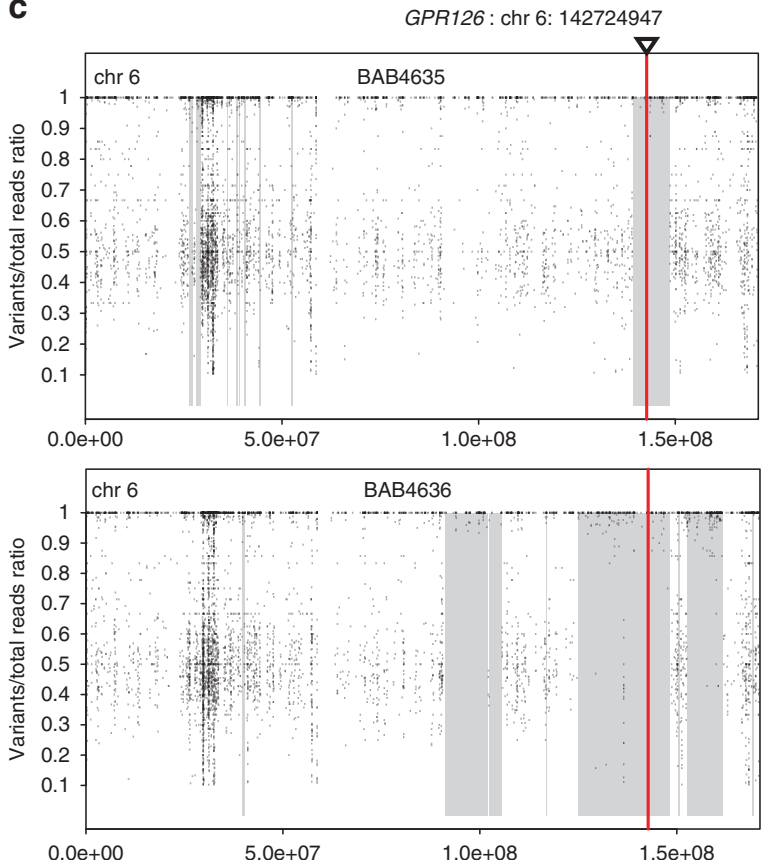

。
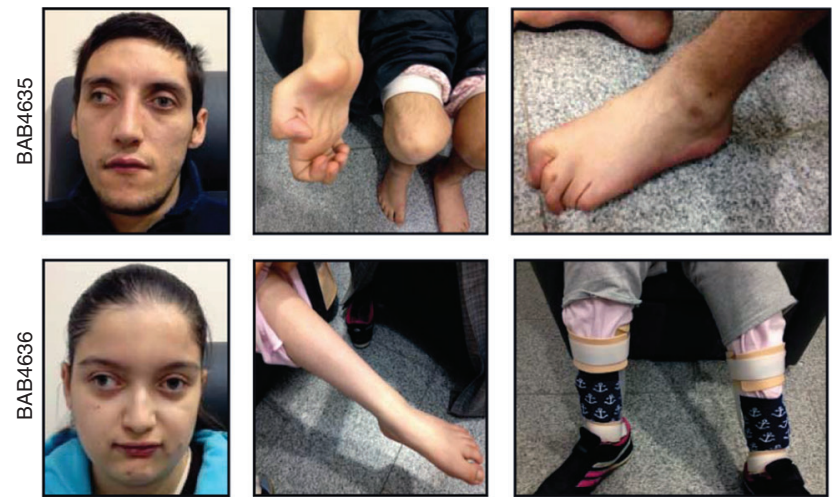

d
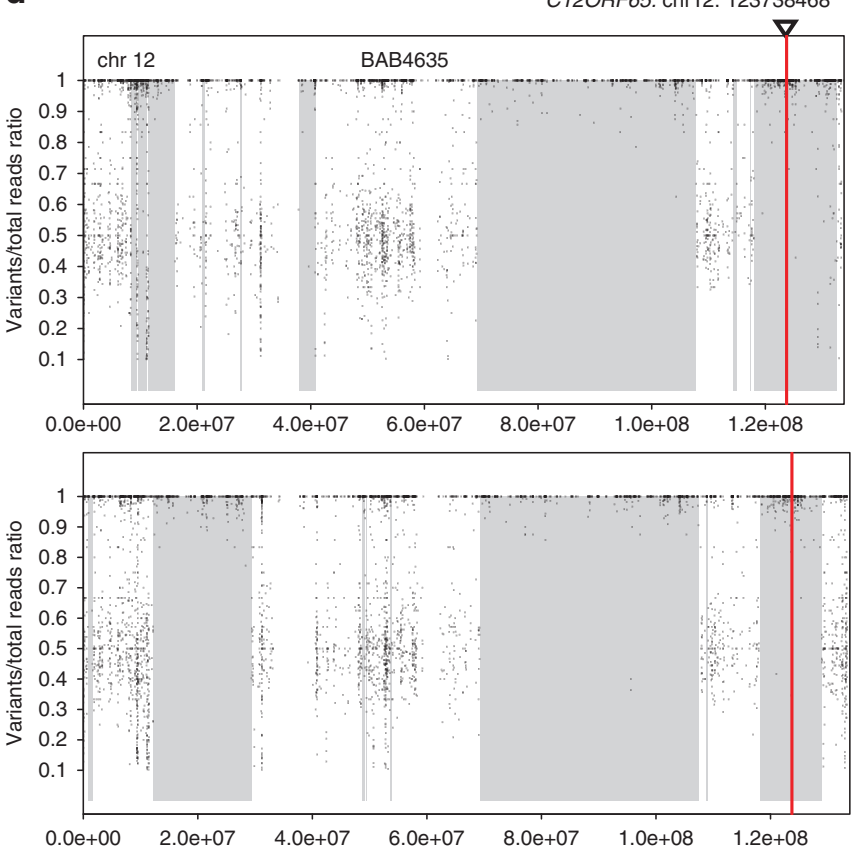

Figure 4 Absence of heterozygosity mediates mutational burden at more than one locus. (a) Pedigree structure of family HOU1857 and segregation of variants identified in C12orf65 and GPR126. (b) Both siblings had esotropia, progressive sensorimotor polyneuropathy with impaired walking and resultant contractures and deformities in their extremities. (c) B-allele frequency calculated from exome variant data demonstrates several regions of absence of heterozygosity $(\mathrm{AOH})$ within chromosome 6, marked by gray zones. Variants in GPR126 (red line) are located in a region of AOH. (d) B-allele frequency calculated from exome variant data demonstrates several regions of AOH within chromosome 12 , marked by gray zones. Variants in C12ORF65 (red line) are located in a region of $\mathrm{AOH}$. 
sometimes third molecular diagnosis identified in a known disease gene, there were a total of 10 variants in four families found within regions of absence of heterozygosity ( $\mathrm{AOH})$, determined based on the calculated B-allele frequency from exome variant data using BafCalculator (https://github.com/ BCM-Lupskilab/BafCalculator) (Supplementary Table S2 online). ${ }^{31}$ The calculated total $\mathrm{AOH}$ for probands of each of the 19 cases with phenotypic expansion ranged from 62.0 to $542.2 \mathrm{Mb}$, and this was not significantly different from that of the studied brain malformation cohort (128 original cases), which ranged from 28.3 to $866.7 \mathrm{Mb}$. Although the relatively small number of cases with phenotypic expansion and/or multilocus variation was not enriched for $\mathrm{AOH}$, the studied brain malformation cohort did demonstrate an enrichment for $\mathrm{AOH}$ compared with all other Turkish individuals in the CMG, and the Turkish cases within the CMG ( 1,000 exomes) were similarly enriched for $\mathrm{AOH}$ compared with non-Turkish individuals in our local CMG exome variant database $(\sim 6,000$ exomes) (Supplementary Figures S1, S2 online).

The utility of $\mathrm{AOH}$ for the study of multilocus variation is perhaps best illustrated by family HOU 1838 for whom there are biallelic variants at two distinct loci, each with evidence that they contribute to the phenotype, and each within the same 44.8- $\mathrm{Mb}$ region of $\mathrm{AOH}$ on chromosome 1 (Figure 3). BAB4474, the proband of HOU1838, was initially described as having a novel pathogenic variant in the gene AP4B1, providing a molecular diagnosis of spastic paraplegia 47, autosomal recessive (OMIM 614066). This diagnosis was consistent with the observed phenotypes of developmental delay/intellectual disability, microcephaly, and agenesis of the corpus callosum, as well as the segregation pattern observed in the pedigree. Extensive reanalysis of the WES data demonstrated additional pathogenic variation at AMPD2, providing etiologic molecular diagnoses potentially explaining the pontocerebellar hypoplasia, thin retina, and protruding tongue (pontocerebellar hypoplasia 9, OMIM 615809 associated with pathogenic variants in $A M P D 2$, Figure 3). Notably, a homozygous splice site variant in $\mathrm{NOTCH} 2$ (c.4512-3T > G) could be consistent with the features of cholelithiasis, thin retina, atrial septal defect, and hirsutism (Alagille syndrome, OMIM 610205 due to variants in NOTCH2); however, the zygosity of the identified variant is not consistent with the autosomal dominant inheritance observed with Alagille syndrome, and we propose that the potential for this locus to be involved in both autosomal recessive and dominant modes of disease trait inheritance requires further exploration. ${ }^{32,33}$

In family HOU1857, siblings BAB4635 and BAB4636 were found to have potentially etiologic variants at two unlinked loci within regions of $\mathrm{AOH}$ (Figure 4). Both siblings presented with agenesis of the corpus callosum, optic atrophy, sensorimotor neuropathy, distal contractures, and a cardiac valve anomaly. After the initial identification of variants in C12ORF65 (combined oxidative phosphorylation deficiency 7, OMIM 613559), homozygous variants in GPR126 (lethal congenital contracture syndrome, OMIM 616503) were identified as causative of the expanded phenotype (Figure 4a,b). Both the C12ORF65 and GPR126 variants are located in distinct but overlapping regions of $\mathrm{AOH}$ on chromosome 12 and 6: C12ORF65 within a region of $11.3 \mathrm{Mb}$ (BAB4635) and $11.0 \mathrm{Mb}$ (BAB4636); GPR126 within a region of $9.5 \mathrm{Mb}$ (BAB4635) and $12.1 \mathrm{Mb}$ (BAB4636, Figure 4c,d).

\section{DISCUSSION}

The availability of WES has enabled a genomic interrogation of phenotypes in a hypothesis-free manner, leading to increasing discoveries of both phenotypic expansion associated with a single genomic locus, and blended phenotypes resulting from multilocus variation. To investigate whether cases of apparent phenotypic expansion may have an increased frequency of multilocus variation, we focused on a cohort of well-characterized neurodevelopmental phenotypes, a subset of which were previously categorized as having phenotypic expansion, occurring in the setting of a high rate of reported consanguinity. ${ }^{20}$ This strategy enabled a focus on predominantly recessive disease and greater likelihood of shared phenotypes amongst siblings with similar genetic backgrounds resulting from identity by descent. ${ }^{30}$ We found that $31.6 \%(6 / 19)$ of cases characterized as demonstrating "phenotypic expansion" actually represent blended phenotypes $^{8}$ with more than one molecular diagnosis, compared with $2.3 \%(2 / 87)$ of cases not characterized as demonstrating phenotypic expansion (two-tailed Fisher's exact $p=0.0004$ ).

Such clinical cases are difficult to recognize as potential dual molecular diagnoses, because blended phenotypes may present as phenotypic expansion, more severe disease along a spectrum, or an apparently novel disease. ${ }^{8}$ Our findings suggest that cases of apparent phenotypic expansion are more likely to present with a blending of distinct disease phenotypes due to multiple molecular diagnoses. We further demonstrate that cases that do not have apparent phenotypic expression can also result from multilocus variation, and may present as a manifestation of more severe disease. Considering this neurodevelopmental cohort in its entirety, which includes 3 cases with multilocus variation reported in the initial study, ${ }^{20}$ a total of 108 cases have one or more molecular diagnoses, of which 13 cases $(12.0 \%, 13 / 108)$ have two or more molecular diagnoses resulting from known or potential novel disease genes, underscoring the frequency with which multiple molecular diagnoses can occur. Notably, the overall rate of multilocus variation in this selected cohort $(12.0 \%)$ is greater than the $4.9 \%$ reported in a diagnostic cohort of mixed phenotypes from an outbred population; ${ }^{8}$ this observation may reflect the genomics of the Turkish population, the high rate of consanguinity, and/or the studied phenotype. As the field moves toward study of oligogenic disease and multilocus pathogenic variation, we will rely heavily on clinical expertise to untangle the relationships between phenotypic expression observed in the clinic and molecular diagnoses identified by genome-wide assays. 
Intrafamilial variability enables a dissection of genotypephenotype correlation

The interplay among genomic variants at more than one locus and the resulting impact on phenotypic expression can be difficult to predict, and a genotype-forward approach to analysis of phenotypic expression is limited by the rarity of each condition, and combination of conditions, studied. Here, we report two families with intrafamilial clinical variability that was ultimately explained by genotypic variability. In family HOU2337, we identified extremely rare and potentially deleterious homozygous variants in both MED17 and SPG7 genes in patient BAB6228, in addition to a hemizygous mutation in the BCOR gene that he shares with his less severely affected brother (Figure 2). Similarly, in family HOU2293, we identified variants in ALG8 and CLN5 in BAB3640, who additionally shared a homozygous MCPH1 variant with her less severely affected sibling (Figure 1). In both families, the expanded phenotype observed in the probands was in fact explained by variation at additional loci not present in their less severely affected siblings. These cases demonstrate the importance of a thorough clinical history and examination of all affected family members. Genomic analysis in families with more than one affected individual allows a dissection of phenotypes and phenotypic severity attributed to variation at each identified locus within the context of a shared genetic background.

\section{$\mathrm{AOH}-$ mediated recessive mutation burden as a tool}

In both families described above, $\mathrm{AOH}$ contributed to a recessive mutation burden, with total $\mathrm{AOH}$, as determined from the calculated B-allele frequency from exome variant data $^{31}$ measuring $509.0 \mathrm{Mb}$ in BAB6228 and 493.1 Mb in BAB3640 (Supplementary Table S3 online). In 14 known or potential autosomal recessive loci identified in this cohort, 11 loci had variants that were homozygous and located within regions of $\mathrm{AOH}$. This finding is particularly striking for patient $\mathrm{BAB} 4474$, for whom analysis revealed rare, homozygous variants in three genes within a single region of $44.8 \mathrm{Mb} \mathrm{AOH}$ : AP4B1 (OMIM 614066), AMPD2, and a potential contribution from NOTCH2 (OMIM 610205, Figure 3, Table 1). Although Alagille syndrome has previously been described as an autosomal dominant condition, BAB4474 is homozygous for the identified variant, which was inherited from apparently unaffected parents. Several examples of genes for which both mono- and biallelic variation lead to disease have been previously described, ${ }^{32-35}$ and we propose that this NOTCH2 variant may represent a mild or hypomorphic allele for which biallelic variation is required for phenotypic expression.

We also describe a family (HOU1857) for which $\mathrm{AOH}-$ mediated recessive mutation burden involves two causative loci located within unlinked regions of $\mathrm{AOH}$ (Figure 4). In this case, the C12ORF65 variant (c.248delT; p.V83fs; spastic paraplegia 55, autosomal recessive OMIM 615035) explained the majority of the clinical features including intellectual disability, optic atrophy, agenesis of the corpus callosum, sensorimotor neuropathy, and contractures (Figure 4, Table 1). A second locus, GPR126, has been shown to be associated with Schwann cell and peripheral nerve development and myelination. We propose contribution of the homozygous GPR126 variant (chr12:123738468_GT > G; c.1880A > G; p.K627R) at this locus to the neuropathy and contractures observed in this family (Figure 4c). ${ }^{36,37}$ In addition, the role of Gpr126 in cardiovascular development has been identified in several animal model studies and may be consistent with the cardiac finding of valvular anomaly in this family. ${ }^{38}$ While total $\mathrm{AOH}$ was not significantly greater in cases with multilocus variation and/or phenotypic expansion compared to the remainder of the neurodevelopmental cohort (Supplementary Figures S1, S2 online), the cohort demonstrated enrichment for $\mathrm{AOH}$ compared with other Turkish individuals and non-Turkish individuals within our local exome database. The burden of homozygous variants identified in regions of $\mathrm{AOH}$ in this cohort demonstrates that $\mathrm{AOH}$-driven recessive disease is one genetic mechanism leading to multiple molecular diagnoses and mutational burden in a patient.

\section{Candidate novel genes}

We propose two novel candidate disease genes, each with the potential to impact our understanding of the pathophysiology of neurologic disease and perhaps delineate a precision medicine-guided pathway to potential therapeutic interventional strategies. In HOU2019, a rare variant in SRR was identified as contributing to the frontotemporal dementia phenotype in the proband (BAB5368), affected mother (BAB5369), and maternal uncle (BAB5371). SRR is a serine racemase that impacts levels of $\mathrm{L}^{-}$and $\mathrm{D}$-serine in the brain, and is thought to be important for glutamatergic neurotransmission, which has been proposed to be involved in the development of complex neurodegenerative disease, particularly Alzheimer disease. ${ }^{25}$ We propose that SRR missense variation results in a gain-of-function allele affecting glutamatergic neurotransmission and resulting in excitatory neurotoxicity and dementia. Additionally, the role of $S R R$ in this phenotype suggests that therapy aimed at modification of glutamatergic neurotransmission may be of potential clinical benefit. In the proband of HOU2128, a rare variant in MAGI3 was identified and may provide both a molecular explanation for the Aicardi phenotype as well as the observed hemihypertrophy. Hemihypertrophy and overgrowth syndromes can be associated with variation in PTEN, and MAGI3 has been demonstrated to impact PTEN regulation of AKT activity. ${ }^{29}$ These examples demonstrate that reanalysis of cases characterized as phenotypic expansion can lead to potential novel candidate disease gene discovery and potentially inform the molecular pathophysiology of disease.

\section{Conclusion}

In conclusion, each genome is truly "personal" and phenotypic expression, what the clinician observes, can be impacted by variation at more than one locus, often resulting in more than 
one molecular diagnosis. ${ }^{8}$ In some cases, phenotypic expansion may be a hallmark of a blended phenotype resulting from multilocus variation. This variation can be driven by the genetic substructure of the family, clan, or population (i.e., isolated, consanguinity), leading to $\mathrm{AOH}$-mediated recessive disease burden. Implementation of WES has enabled genomic medicine to move from individual study of single-gene Mendelian disorders to a more comprehensive understanding of the interplay of genetic variation at more than one locus and resulting phenotypic expression. These findings further emphasize the important role of the clinician and robust physical examination and clinical characterization of each patient to recognize blended phenotypes due to multilocus variation. Such blended phenotypes may present clinically as distinct phenotypes manifesting as apparent phenotypic expansion associated with the first identified locus, or overlapping phenotypes that may manifest as an apparent worsening of disease severity.

\section{SUPPLEMENTARY MATERIAL}

Supplementary material is linked to the online version of the paper at http://www.nature.com/gim

\section{ACKNOWLEDGMENTS}

J.E.P. was supported by a Chao Physician-Scientist Award through the Ting Tsung and Wei Fong Chao Foundation and K08 HG008986 through the National Human Genome Research Institute. This work was supported in part by grants UM1 HG006542 (J.R.L.) from the National Human Genome Research Institute/National Heart, Lung, and Blood Institute to the Baylor Hopkins Center for Mendelian Genomics, R01 NS058529 and R35 NS105078 (J.R.L.) from the National Institute of Neurological Disorders and Stroke, and U54-HG003273 (R.A.G.) from the National Human Genome Research Institute.

\section{DISCLOSURE}

Baylor College of Medicine and Miraca Holdings have formed a joint venture with shared ownership and governance of Baylor Genetics, formerly the Baylor Miraca Genetics Laboratories, which performs clinical exome sequencing. J.R.L. serves on the Scientific Advisory Board of Baylor Genetics. J.R.L. has stock ownership in 23andMe, is a paid consultant for Regeneron Pharmaceuticals, has stock options in Lasergen, and is a coinventor on multiple US and European patents related to molecular diagnostics for inherited neuropathies, eye diseases, and bacterial genomic fingerprinting. The other authors declare no conflict of interest.

\section{REFERENCES}

1. Fitzsimmons JS \& Guilbert PR. Spastic paraplegia associated with brachydactyly and cone shaped epiphyses. J Med Genet. 1987;24: 702-705.

2. Hennekam RC. Spastic paraplegia, dysarthria, brachydactyly, and cone shaped epiphyses: confirmation of the Fitzsimmons syndrome. J Med Genet. 1994;31:251-252.

3. Armour CM, Humphreys P, Hennekam RC \& Boycott KM. Fitzsimmons syndrome: spastic paraplegia, brachydactyly and cognitive impairment. Am J Med Genet A. 2009;149A:2254-2257.
4. Armour CM, Smith A, Hartley T, et al. Syndrome disintegration: Exome sequencing reveals that Fitzsimmons syndrome is a co-occurrence of multiple events. Am J Med Genet A. 2016;170:1820-1825.

5. Shroyer NF, Lewis RA, Yatsenko AN \& Lupski JR. Null missense $A B C R$ $(A B C A 4)$ mutations in a family with stargardt disease and retinitis pigmentosa. Invest Ophthalmol Vis Sci. 2001;42:2757-2761.

6. Lee $\mathrm{W}, \mathrm{Xie} Y$, Zernant J, et al. Complex inheritance of $A B C A 4$ disease: four mutations in a family with multiple macular phenotypes. Hum Genet. 2016;135:9-19.

7. Jehee FS, de Oliveira VT, Gurgel-Giannetti J, et al. Dual molecular diagnosis contributes to atypical Prader-Willi phenotype in monozygotic twins. Am J Med Genet A. 2017;173:2451-2455.

8. Posey JE, Harel T, Liu P, et al. Resolution of Disease Phenotypes Resulting from Multilocus Genomic Variation. N Engl J Med. 2017;376:21-31.

9. Cahill KM \& Ley $A B$. Favism and thalassemia minor in a pregnant woman. JAMA. 1962;180:119-121.

10. Fraser GR, Stamatoyannopoulos G, Kattamis C, et al. Thalassemias, abnormal hemoglobins and glucose-6-phosphate dehydrogenase deficiency in the Arta area of Greece: diagnostic and genetic aspects of complete village studies. Ann NY Acad Sci. 1964;119:415-435.

11. Girirajan S, Rosenfeld JA, Coe BP, et al. Phenotypic heterogeneity of genomic disorders and rare copy-number variants. $N$ Engl J Med. 2012:367:1321-1331.

12. Farwell KD, Shahmirzadi L, El-Khechen D, et al. Enhanced utility of familycentered diagnostic exome sequencing with inheritance model-based analysis: results from 500 unselected families with undiagnosed genetic conditions. Genet Med. 2015;17:578-586.

13. Yang $Y$, Muzny DM, Xia $F$, et al. Molecular findings among patients referred for clinical whole-exome sequencing. JAMA. 2014;312: 1870-1879.

14. Yang Y, Muzny DM, Reid JG, et al. Clinical whole-exome sequencing for the diagnosis of Mendelian disorders. N Engl J Med. 2013;369: 1502-1511.

15. Retterer K, Juusola J, Cho MT, et al. Clinical application of whole-exome sequencing across clinical indications. Genet Med. 2016;18:696-704.

16. Posey JE, Rosenfeld JA, James RA, et al. Molecular diagnostic experience of whole-exome sequencing in adult patients. Genet Med. 2016;18: 678-685.

17. Balci TB, Hartley $T, X i Y$, et al. Debunking Occam's razor: diagnosing multiple genetic diseases in families by whole-exome sequencing. Clin Genet. 2017;92:281-289.

18. Tarailo-Graovac M, Shyr C, Ross CJ, et al. Exome sequencing and the management of neurometabolic disorders. N Engl J Med. 2016;374: 2246-2255.

19. Chong JX, Buckingham KJ, Jhangiani SN, et al. The genetic basis of Mendelian phenotypes: discoveries, challenges, and opportunities. Am J Hum Genet. 2015;97:199-215.

20. Karaca E, Harel T, Pehlivan D, et al. Genes that affect brain structure and function identified by rare variant analyses of Mendelian neurologic disease. Neuron. 2015;88:499-513.

21. Xia F, Bainbridge MN, Tan TY, et al. De novo truncating mutations in AHDC1 in individuals with syndromic expressive language delay, hypotonia, and sleep apnea. Am J Hum Genet. 2014;94:784-789.

22. Jiang $Y$, Wangler $M, M c G u i r e ~ A$, et al. The phenotypic spectrum of XiaGibbs syndrome. Am J Med Genet $A$ (in press).

23. Hock M, Wegleiter K, Ralser E, et al. ALG8-CDG: novel patients and review of the literature. Orphanet J Rare Dis. 2015;10:73.

24. Savukoski M, Klockars T, Holmberg V, Santavuori P, Lander ES \& Peltonen L. CLN5, a novel gene encoding a putative transmembrane protein mutated in Finnish variant late infantile neuronal ceroid lipofuscinosis. Nat Genet. 1998;19:286-288.

25. Inoue R, Hashimoto K, Harai T \& Mori H. NMDA- and beta-amyloid1-42induced neurotoxicity is attenuated in serine racemase knock-out mice. J Neurosci. 2008;28:14486-14491.

26. Franklin JL, Yoshiura K, Dempsey PJ, et al. Identification of MAGI-3 as a transforming growth factor-alpha tail binding protein. Exp Cell Res. 2005;303:457-470

27. Hamdan FF, Gauthier J, Araki Y, et al. Excess of de novo deleterious mutations in genes associated with glutamatergic systems in nonsyndromic intellectual disability. Am J Hum Genet. 2011;88: 306-316.

28. Marshall CR, Young EJ, Pani AM, et al. Infantile spasms is associated with deletion of the MAGI2 gene on chromosome 7q11.23-q21.11. Am J Hum Genet. 2008;83:106-111. 
29. Wu Y, Dowbenko D, Spencer $S$, et al. Interaction of the tumor suppressor PTEN/MMAC with a PDZ domain of MAGI3, a novel membraneassociated guanylate kinase. J Biol Chem. 2000;275:21477-21485.

30. Lupski JR, Belmont JW, Boerwinkle E \& Gibbs RA. Clan genomics and the complex architecture of human disease. Cell. 2011;147:32-43.

31. Gambin T, Coban Akdemir Z, Yuan B, et al. Homozygous and hemizygous CNV detection from exome sequencing data in a Mendelian disease cohort. Nucleic Acids Res. 2017;45:1633-1648.

32. Harel T, Yesil G, Bayram Y, et al. Monoallelic and biallelic variants in EMC1 identified in individuals with global developmental delay, hypotonia, scoliosis, and cerebellar atrophy. Am J Hum Genet. 2016;98:562-570.

33. Harel T, Yoon WH, Garone $C$, et al. Recurrent de novo and biallelic variation of $A T A D 3 A$, encoding a mitochondrial membrane protein, results in distinct neurological syndromes. Am J Hum Genet. 2016;99: 831-845.
34. Monies D, Maddirevula S, Kurdi W, et al. Autozygosity reveals recessive mutations and novel mechanisms in dominant genes: implications in variant interpretation. Genet Med. 2017;19:1144-1150.

35. Rainger J, Pehlivan D, Johansson S, et al. Monoallelic and biallelic mutations in MAB21L2 cause a spectrum of major eye malformations. Am J Hum Genet. 2014;94:915-923.

36. Mogha A, Benesh AE, Patra C, et al. Gpr126 functions in Schwann cells to control differentiation and myelination via G-protein activation. J Neurosci. 2013;33:17976-17985.

37. Ravenscroft G, Nolent F, Rajagopalan S, et al. Mutations of GPR126 are responsible for severe arthrogryposis multiplex congenita. Am J Hum Genet. 2015;96:955-961.

38. Cui H, Wang $Y$, Huang $H$, et al. GPR126 protein regulates developmental and pathological angiogenesis through modulation of VEGFR2 receptor signaling. J Biol Chem. 2014;289:34871-34885. 